\title{
Some Formulae of n-Norms and Their Identicalness in a Hilbert Space
}

\author{
M. P. Singh*, S. Romen Meitei \\ Manipur University, Canchipur, Imphal, Manipur, India. \\ * Corresponding author. Tel.: +919612950576; email: mpremjitmu@gmail.com \\ Manuscript submitted October 13, 2015; accepted December 12, 2015. \\ doi: 10.17706/ijapm.2016.6.1.25-30
}

\begin{abstract}
In this paper, we discuss the concept of n-normed spaces and generalize a formulae of n-norm. Further we prove the equality of seven formulae of n-norms on a Hilbert space and eight formulae of n-norms on a separable Hilbert space. An alternative formula of n-norm on the dual of an n-normed space is introduced. Also, we show its equality with two alternative formulae.
\end{abstract}

2010 Mathematics Subject Classification: 46B20, 46C05, 46C15, 46B99, 46C99.

Key words: Dual space, Hilbert space, inner product, norm, n-norm, separable space.

\section{Introduction}

Let $X$ be a real vector space with $\operatorname{dim} X \geqslant n$, where $n$ is a positive integer. A real valued function $\|, \ldots, .,: X^{n} \rightarrow \mathbb{R}$ is called an $n$-norm on $X$ if the following conditions hold:

1) $\left\|x_{1}, \ldots, x_{n}\right\|=0$ iff $x_{1}, \ldots, x_{n}$ are linearly dependent.

2) $\left\|x_{1}, \ldots, x_{n}\right\|$ is invariant under permutations of $x_{1}, \ldots, x_{n}$.

3) $\left\|\alpha x_{1}, x_{2}, \ldots, x_{n}\right\|=\mid \alpha\left\|x_{1}, \ldots, x_{n}\right\|$ for any $\alpha \in \mathbb{R}$.

4) $\left\|x_{0}+x_{1}, x_{2}, \ldots, x_{n}\right\| \leq\left\|x_{0}, \ldots, x_{n}\right\|+\left\|x_{1}, \ldots, x_{n}\right\|$ for all $x_{0}, x_{1}, \ldots, x_{n} \in X$.

The pair $(X, \|, \ldots, n)$ is called an $\mathrm{n}$-normed space. An $\mathrm{n}$-norm is always non-negative. The combination of conditions (3) and (4) above gives the non-negativity of an n-norm. If $X$ is an n-normed space with dual $X^{\prime}$, the following formula (as formulated by "Gähler [1]").

$$
\left\|x_{1}, \ldots, x_{n}\right\|^{G}=\operatorname{Sup}_{f_{j} \in X^{\prime},\left\|f_{j}\right\| \leq 1}\left|\begin{array}{ccc}
f_{1}\left(x_{1}\right) & \cdots & f_{n}\left(x_{1}\right) \\
\vdots & \ddots & \vdots \\
f_{1}\left(x_{n}\right) & \cdots & f_{n}\left(x_{n}\right)
\end{array}\right|
$$

defines an n-norm on $x$.

If $X$ is equipped with an inner product $\langle. .$.$\rangle , we can define the standard n-norm on X$ by $\left\|x_{1}, \ldots, x_{n}\right\|^{S}=\sqrt{\operatorname{det}\left[\left\langle x_{i}, x_{j}\right\rangle\right]}$.

Note that the value of $\left\|x_{1}, \ldots, x_{n}\right\|^{s}$ represents the volume of n-dimensional parallelepiped spanned by $x_{1}, \ldots, x_{n}$. Let $x$ be a Hilbert space with dual $x^{\prime}$.Then Gähler's formula on $x$ becomes $\left\|x_{1}, \ldots, x_{n}\right\|^{G}=\underset{y_{j} \in x|y| y \mid \leqslant 1}{\operatorname{Sup}} \operatorname{det}\left[\left\langle x_{i}, y_{j}\right\rangle\right]$. 
Also the function

$$
\left\|x_{1}, \ldots, x_{n}\right\|^{D}=\operatorname{Sup}_{y_{j} \in X,\|\|_{1}, \ldots, y_{n} f_{1} \leq 1}\left|\begin{array}{ccc}
\left\langle x_{1}, y_{1}\right\rangle & \cdots & \left\langle x_{1}, y_{n}\right\rangle \\
\vdots & \ddots & \vdots \\
\left\langle x_{n}, y_{1}\right\rangle & \cdots & \left\langle x_{n}, y_{n}\right\rangle
\end{array}\right|
$$

defines an n-norm on a Hilbert space $x$. Then $\|, \ldots,\|^{G}$ and $\quad\|, \ldots,\|^{D}$ are identical on a Hilbert space $\quad x \quad[2]$.

If $x$ is a separable Hilbert space and $\left\{e_{1}, e_{2}, \ldots\right\}$ is a complete orthonormal set in $x$, we can define an n-norm on $x$ by

$$
\left\|x_{1}, \ldots, x_{n}\right\|_{2}=\left[\frac{1}{n !} \sum_{j_{1}} \cdots \sum_{j_{n}} \mid \operatorname{det}\left[\alpha_{i_{k}}\right]^{2}\right]^{\frac{1}{2}}
$$

where $\alpha_{i j}=\left\langle x_{i}, e_{j}\right\rangle[2],[3]$.

Further, the function $\left\|x_{1}, \ldots, x_{n}\right\|^{E}=\operatorname{Sup}_{y_{j} \in X,\left|y_{n}, \ldots, y_{n}\right|^{s}=1}\left|\begin{array}{ccc}\left\langle x_{1}, y_{1}\right\rangle & \cdots & \left\langle x_{1}, y_{n}\right\rangle \\ \vdots & \ddots & \vdots \\ \left\langle x_{n}, y_{1}\right\rangle & \cdots & \left\langle x_{n}, y_{n}\right\rangle\end{array}\right|$ defines an n-norm on a Hilbert space and the function

$$
\left\|x_{1}, \ldots, x_{n}\right\|^{r}=\operatorname{Sup}_{f_{j} \in X^{\prime}|f(f)|=1}\left|\begin{array}{ccc}
f_{1}\left(x_{1}\right) & \cdots & f_{n}\left(x_{1}\right) \\
\vdots & \ddots & \vdots \\
f_{1}\left(x_{n}\right) & \cdots & f_{n}\left(x_{n}\right)
\end{array}\right|
$$

defines an n-norm on a normed space $X$ with dual $X^{\prime}$ [4].

If $x$ is a Hilbert space, $\|, \ldots, \ldots,\|^{r}$ becomes $\left\|x_{1}, \ldots, x_{n}\right\|^{r}=\sup _{y_{j} \in x,\|,\|=1} \operatorname{det}\left[\left\langle x_{i}, y_{j}\right\rangle\right]$. Then $\|, \ldots, .,\|^{D},\|, \ldots,\|^{E},\|, \ldots,\|^{G},\|, \ldots,\|^{r}$ and $\|, \ldots,\|^{S}$ are identical on a Hilbert space and they are identical with $\|, \ldots, \ldots,\|_{2}$ on a separable Hilbert space.

$$
\text { Also, }\left\|f_{1}, \ldots, f_{n}\right\|^{\prime}=\operatorname{Sup}_{x_{i} \in X, \|_{1}, \ldots, x_{n} \mid \leqslant 1}\left|\begin{array}{ccc}
f_{1}\left(x_{1}\right) & \cdots & f_{n}\left(x_{1}\right) \\
\vdots & \ddots & \vdots \\
f_{1}\left(x_{n}\right) & \cdots & f_{n}\left(x_{n}\right)
\end{array}\right| \text { and }\left\|f_{1}, \ldots, f_{n}\right\|_{1}^{\prime}=\operatorname{Sup}_{x_{i} \in X, x_{1}, \ldots, x_{n} \mid=1}\left|\begin{array}{ccc}
f_{1}\left(x_{1}\right) & \cdots & f_{n}\left(x_{1}\right) \\
\vdots & \ddots & \vdots \\
f_{1}\left(x_{n}\right) & \cdots & f_{n}\left(x_{n}\right)
\end{array}\right|
$$

are identical n-norms on $x^{\prime}$, the dual of an n-normed space $x$ [4].

The theory of 2-normed spaces and n-normed spaces were initially developed by Gähler [1], [5]-[7] in the 1960's. Recent works and related works can be found in [2], [3], [8]-[10]. The most recent work can be seen in [4]. Our interest here is to study alternative formulae of n-norms especially in a Hilbert space. The alternative formulae are identical with the n-norms mentioned above. In the last part we study the equality of three n-norms defined on the dual space of an n-normed space.

\section{Generalization of an $\mathbf{n}$-Norm}

Let $x$ be a real vector space with $\operatorname{dim} x \geq n$ equipped with an inner product $\langle. .$.$\rangle . Then the function$

$$
\left\|x_{1}, \ldots, x_{n}\right\|^{R}=A b s\left(\begin{array}{ccc}
\left\langle x_{1}, y_{1}\right\rangle & \cdots & \left\langle x_{1}, y_{n}\right\rangle \\
\vdots & \ddots & \vdots \\
\left\langle x_{n}, y_{1}\right\rangle & \cdots & \left\langle x_{n}, y_{n}\right\rangle
\end{array}\right)
$$

defines an n-norm on $x$ for fixed linearly independent $\mathrm{n}$ elements $y_{1}, \ldots, y_{n} \in X \quad[4]$.

The following proposition is the generalization of the above proposition. 
Proposition 2.1. Let $X$ be a normed space of $\operatorname{dim} X \geq n$ with dual $X^{\prime}$. Then the function

$$
\left.\left\|x_{1}, \ldots, x_{n}\right\|=A b s\left(\begin{array}{ccc}
\mid f_{1}\left(x_{1}\right) & \cdots & f_{n}\left(x_{1}\right) \\
\vdots & \ddots & \vdots \\
f_{1}\left(x_{n}\right) & \cdots & f_{n}\left(x_{n}\right)
\end{array}\right)\right)
$$

defines an n-norm on $X$ for fixed linearly independent $\mathrm{n}$ funtionals $f_{1}, f_{2}, \ldots, f_{n} \in X^{\prime}$.

Proof: (i) It is easy to show that $x_{1}, \ldots, x_{n}$ are linearly dependent iff $\left\|x_{1}, \ldots, x_{n}\right\|=0$.

(ii) The absolute value of a determinant remains invariant under the interchange of rows (or columns). $\Rightarrow\left\|x_{1}, \ldots, x_{n}\right\|$ is invariant under the permutations of $x_{1}, \ldots, x_{n}$.

(iii) $\forall \alpha \in \mathbb{R}$,

$$
\begin{gathered}
\left\|\alpha x_{1}, \ldots, x_{n}\right\|=A b s\left(\left|\begin{array}{ccc}
f_{1}\left(\alpha x_{1}\right) & \cdots & f_{n}\left(\alpha x_{1}\right) \\
\vdots & \ddots & \vdots \\
f_{1}\left(x_{n}\right) & \cdots & f_{n}\left(x_{n}\right)
\end{array}\right|\right) \\
=A b s\left(\left|\begin{array}{ccc}
\alpha f_{1}\left(x_{1}\right) & \cdots & \alpha f_{n}\left(x_{1}\right) \\
\vdots & \ddots & \vdots \\
f_{1}\left(x_{n}\right) & \cdots & f_{n}\left(x_{n}\right)
\end{array}\right|\right)\left(\because f_{i}^{\prime} \text { s are linear }\right) \\
=|\alpha| A b s\left(\mid \begin{array}{ccc}
f_{1}\left(x_{1}\right) & \cdots & f_{n}\left(x_{1}\right) \\
\vdots & \ddots & \vdots \\
f_{1}\left(x_{n}\right) & \cdots & f_{n}\left(x_{n}\right)
\end{array}\right) \\
=\mid \alpha\left\|x_{1}, \ldots, x_{n}\right\|
\end{gathered}
$$

(iv) For $x_{0}, x_{1}, \ldots, x_{n} \in X$,

$$
\begin{gathered}
\left|\begin{array}{ccc}
f_{1}\left(x_{0}+x_{1}\right) & \cdots & f_{n}\left(x_{0}+x_{1}\right) \\
\vdots & \ddots & \vdots \\
f_{1}\left(x_{n}\right) & \cdots & f_{n}\left(x_{n}\right)
\end{array}\right|=\left|\begin{array}{ccc}
f_{1}\left(x_{0}\right) & \cdots & f_{n}\left(x_{0}\right) \\
\vdots & \ddots & \vdots \\
f_{1}\left(x_{n}\right) & \cdots & f_{n}\left(x_{n}\right)
\end{array}\right|+\left|\begin{array}{ccc}
f_{1}\left(x_{1}\right) & \cdots & f_{n}\left(x_{1}\right) \\
\vdots & \ddots & \vdots \\
f_{1}\left(x_{n}\right) & \cdots & f_{n}\left(x_{n}\right)
\end{array}\right| \\
\Rightarrow A b s\left(\begin{array}{ccc}
\mid f_{1}\left(x_{0}+x_{1}\right) & \cdots & f_{n}\left(x_{0}+x_{1}\right) \\
\vdots & \ddots & \vdots \\
f_{1}\left(x_{n}\right) & \cdots & f_{n}\left(x_{n}\right)
\end{array} \mid\right) \leq A b s\left(\begin{array}{ccc}
\mid f_{1}\left(x_{0}\right) & \cdots & f_{n}\left(x_{0}\right) \\
\vdots & \ddots & \vdots \\
f_{1}\left(x_{n}\right) & \cdots & f_{n}\left(x_{n}\right)
\end{array}\right)+A b s\left(\begin{array}{ccc}
\mid f_{1}\left(x_{1}\right) & \cdots & f_{n}\left(x_{1}\right) \\
\vdots & \ddots & \vdots \\
f_{1}\left(x_{n}\right) & \cdots & f_{n}\left(x_{n}\right)
\end{array}\right) \\
\Rightarrow\left\|x_{0}+x_{1}, \cdots, x_{n}\right\| \leq\left\|x_{0}, \cdots, x_{n}\right\|+\left\|x_{1}, \ldots, x_{n}\right\| .
\end{gathered}
$$

This completes the proof.

Remark: If $X$ is a Hilbert space with dual $X^{\prime}$, the above n-norm $\|, \ldots, .,\|^{\prime}$ becomes $\|, \ldots,\|^{R}$. It follows from:

By Riesz-representation theorem, for each fixed bounded linear functional $f_{j} \in X^{\prime}$, there exists unique $y_{j} \in X$ such that $f_{j}\left(x_{i}\right)=\left\langle x_{i}, y_{j}\right\rangle \&\left\|f_{j}\right\|=\left\|y_{j}\right\|$. Then, $\left\|x_{1}, \ldots, x_{n}\right\|=A b s\left(\begin{array}{ccc}\mid f_{1}\left(x_{1}\right) & \cdots & f_{n}\left(x_{1}\right) \\ \vdots & \ddots & \vdots \\ f_{1}\left(x_{n}\right) & \cdots & f_{n}\left(x_{n}\right)\end{array}\right)$ 
$=A b s\left(\mid \begin{array}{ccc}\left\langle x_{1}, y_{1}\right\rangle & \cdots & \left\langle x_{1}, y_{n}\right\rangle \\ \vdots & \ddots & \vdots \\ \left\langle x_{n}, y_{1}\right\rangle & \cdots & \left\langle x_{n}, y_{n}\right\rangle\end{array}\right)$ for linearly independent $n$ elements $y_{1}, \ldots, y_{n} \quad=\left\|x_{1}, \ldots, x_{n}\right\|^{R}$.

\section{Identicalness of Alternative n-Norms}

Proposition 3.1. The function $\left\|x_{1}, \ldots, x_{n}\right\|^{F}=\operatorname{Sup}_{y_{f} \in X,\left\|y_{1}, \ldots, y_{n}\right\|^{s} \neq 0} \frac{\left|\begin{array}{ccc}\left\langle x_{1}, y_{1}\right\rangle & \cdots & \left\langle x_{1}, y_{n}\right\rangle \\ \vdots & \ddots & \vdots \\ \left\langle x_{n}, y_{1}\right\rangle & \cdots & \left\langle x_{n}, y_{n}\right\rangle\end{array}\right|}{\left\|y_{1}, \ldots, y_{n}\right\|^{S}}$ defines an n-norm on a Hilbert space $X$.

Proof: (i) It is easy to show that $x_{1}, \ldots, x_{n}$ are linearly dependent iff $\left\|x_{1}, \ldots, x_{n}\right\|^{F}=0$.

(ii) By the properties of determinant and definition of supremum, $\left\|x_{1}, \ldots, x_{n}\right\|^{F}$ remains invariant under the permutations of $x_{1}, \ldots, x_{n}$.

(iii) $\forall \alpha \in \mathbb{R}$,

$$
\begin{aligned}
& \left\|\alpha x_{1}, \ldots, x_{n}\right\|^{F}=\operatorname{Sup}_{y_{j} \in X,\left\|y_{1}, \ldots, y_{n}\right\|^{S} \neq 0} \frac{\left|\begin{array}{ccc}
\left\langle\alpha x_{1}, y_{1}\right\rangle & \cdots & \left\langle\alpha x_{1}, y_{n}\right\rangle \\
\vdots & \ddots & \vdots \\
\left\langle x_{n}, y_{1}\right\rangle & \cdots & \left\langle x_{n}, y_{n}\right\rangle
\end{array}\right|}{\left\|y_{1}, \ldots, y_{n}\right\|^{S}} \\
& =\operatorname{Sup}_{y_{j} \in X,\left\|y_{1}, \ldots, y_{n}\right\|^{S} \neq 0} \frac{\left|\begin{array}{ccc}
\alpha\left\langle x_{1}, y_{1}\right\rangle & \cdots & \alpha\left\langle x_{1}, y_{n}\right\rangle \\
\vdots & \ddots & \vdots \\
\left\langle x_{n}, y_{1}\right\rangle & \cdots & \left\langle x_{n}, y_{n}\right\rangle
\end{array}\right|}{\left\|y_{1}, \ldots, y_{n}\right\|^{S}} \\
& =\operatorname{Sup}_{y_{j} \in X,\left\|y_{1}, \ldots, y_{n}\right\|^{\mid} \neq 0} \frac{\left\langle\left\langle\begin{array}{ccc}
\left\langle x_{1}, y_{1}\right\rangle & \cdots & \left\langle x_{1}, y_{n}\right\rangle \\
\vdots & \ddots & \vdots \\
\left\langle x_{n}, y_{1}\right\rangle & \cdots & \left\langle x_{n}, y_{n}\right\rangle
\end{array}\right|\right.}{\left\|y_{1}, \ldots, y_{n}\right\|^{S}}
\end{aligned}
$$

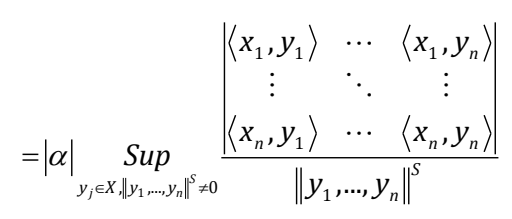

$$
\begin{aligned}
& =\mid \alpha\left\|x_{1}, \ldots, x_{n}\right\|^{F}
\end{aligned}
$$

(iv) For $x_{0}, x_{1}, \ldots, x_{n} \in X$,

$$
\begin{gathered}
\frac{\left|\begin{array}{ccc}
\left\langle x_{0}+x_{1}, y_{1}\right\rangle & \cdots & \left\langle x_{0}+x_{1}, y_{n}\right\rangle \\
\vdots & \ddots & \vdots \\
\left\langle x_{n}, y_{1}\right\rangle & \cdots & \left\langle x_{n}, y_{n}\right\rangle
\end{array}\right|}{\left\|y_{1}, \ldots, y_{n}\right\|^{S}}=\frac{\left|\begin{array}{cccc}
\left\langle x_{0}, y_{1}\right\rangle+\left\langle x_{1}, y_{1}\right\rangle & \cdots & \left\langle x_{0}, y_{n}\right\rangle+\left\langle x_{1}, y_{n}\right\rangle \\
\vdots & \ddots & \vdots \\
\left\langle x_{n}, y_{1}\right\rangle & \cdots & \left\langle x_{n}, y_{n}\right\rangle
\end{array}\right|}{\left\|y_{1}, \ldots, y_{n}\right\|^{S}} \\
=\frac{\left|\begin{array}{ccc}
\left\langle x_{0}, y_{1}\right\rangle & \cdots & \left\langle x_{0}, y_{n}\right\rangle \\
\vdots & \ddots & \vdots \\
\left\langle x_{n}, y_{1}\right\rangle & \cdots & \left\langle x_{n}, y_{n}\right\rangle
\end{array}\right|}{\left\|y_{1}, \ldots, y_{n}\right\|^{S}}+\frac{\left|\begin{array}{ccc}
\left\langle x_{1}, y_{1}\right\rangle & \cdots & \left\langle x_{1}, y_{n}\right\rangle \\
\vdots & \ddots & \vdots \\
\left\langle x_{n}, y_{1}\right\rangle & \cdots & \left\langle x_{n}, y_{n}\right\rangle
\end{array}\right|}{\left\|y_{1}, \ldots, y_{n}\right\|^{S}}
\end{gathered}
$$




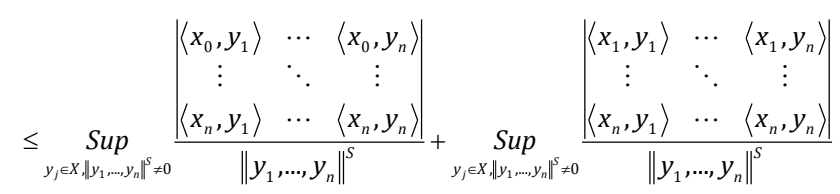

$$
\begin{aligned}
& \Rightarrow \frac{\left|\begin{array}{ccc}
\left\langle x_{0}+x_{1}, y_{1}\right\rangle & \cdots & \left\langle x_{0}+x_{1}, y_{n}\right\rangle \\
\vdots & \ddots & \vdots \\
\left\langle x_{n}, y_{1}\right\rangle & \cdots & \left\langle x_{n}, y_{n}\right\rangle
\end{array}\right|}{\left\|y_{1}, \ldots, y_{n}\right\|^{S}} \leq\left\|x_{0}, x_{2}, \ldots, x_{n}\right\|^{F}+\left\|x_{1}, x_{2}, \ldots, x_{n}\right\|^{F} \text { for all } y_{1}, \ldots, y_{n} \in X \text { with }\left\|y_{1}, \ldots, y_{n}\right\|^{s} \neq 0 . \\
& \Rightarrow\left\|x_{0}+x_{1}, x_{2}, \ldots, x_{n}\right\|^{F} \leq\left\|x_{0}, x_{2}, \ldots, x_{n}\right\|^{F}+\left\|x_{1}, x_{2}, \ldots, x_{n}\right\|^{F} .
\end{aligned}
$$

This completes the proof.

Proposition 3.2. On a Hilbert space $\mathrm{X}$ with $\operatorname{dim} X \geq n$, the two formulae $\|, \ldots,\|^{E}$ and $\|, \ldots,\|^{F}$ are identical. Proof:

$$
\left\|x_{1}, \ldots, x_{n}\right\|^{E}=\operatorname{Sup}_{y_{1} \in X, \mid y_{1}, \ldots, y_{n} \|^{\mid}=1}\left|\begin{array}{cccc}
\left\langle x_{1}, y_{1}\right\rangle & \cdots & \left\langle x_{1}, y_{n}\right\rangle \\
\vdots & \ddots & \vdots \\
\left\langle x_{n}, y_{1}\right\rangle & \cdots & \left\langle x_{n}, y_{n}\right\rangle
\end{array}\right|
$$

And

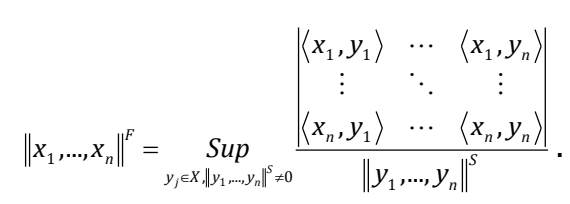

Clearly,

$$
\left\|x_{1}, \ldots, x_{n}\right\|^{E} \leq\left\|x_{1}, \ldots, x_{n}\right\|^{F}
$$

Conversely, we choose $z_{j}=\frac{y_{j}}{\sqrt[n]{\left\|y_{1}, \ldots, y_{n}\right\|^{s}}}=\frac{y_{j}}{a}, a=\sqrt[n]{\mid y_{1}, \ldots, y_{n} \|^{S}} \neq 0$ for $j=1,2, \ldots, n$.

Then,

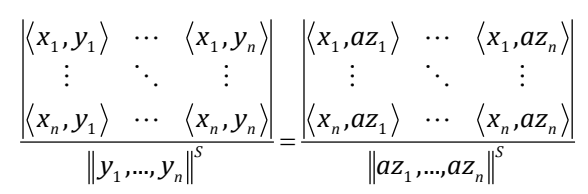

$$
\begin{aligned}
& =\frac{a^{n}\left|\begin{array}{ccc}
\left\langle x_{1}, z_{1}\right\rangle & \cdots & \left\langle x_{1}, z_{n}\right\rangle \\
\vdots & \ddots & \vdots \\
\left\langle x_{n}, z_{1}\right\rangle & \cdots & \left\langle x_{n}, z_{n}\right\rangle
\end{array}\right|}{a^{n}\left\|z_{1}, \ldots, z_{n}\right\|^{s}} \\
& =\left|\begin{array}{ccc}
\left\langle x_{1}, z_{1}\right\rangle & \cdots & \left\langle x_{1}, z_{n}\right\rangle \\
\vdots & \ddots & \vdots \\
\left\langle x_{n}, z_{1}\right\rangle & \cdots & \left\langle x_{n}, z_{n}\right\rangle
\end{array}\right|\left(\because \cdot\left\|z_{1}, \ldots, z_{n}\right\|^{s}=1\right) \\
& \leq \operatorname{Sup}_{z, \in X, \|\left.\right|_{1}, \ldots,\left.z_{n}\right|^{s}=1}\left\langle\begin{array}{ccc}
\left\langle x_{1}, z_{1}\right\rangle & \cdots & \left\langle x_{1}, z_{n}\right\rangle \\
\vdots & \ddots & \vdots \\
\left\langle x_{n}, z_{1}\right\rangle & \cdots & \left\langle x_{n}, z_{n}\right\rangle
\end{array}\right|
\end{aligned}
$$




$$
\begin{gathered}
=\left\|x_{1}, \ldots, x_{n}\right\|^{E} \text { for all } y_{j} \in x \text { with }\left\|y_{1}, \ldots, y_{n}\right\|^{S} \neq 0 . \\
\Rightarrow \operatorname{Sup}_{y_{j} \in X,\left\|y_{1}, \ldots, y_{n}\right\|^{F} \neq 0} \frac{\left|\begin{array}{ccc}
\left\langle x_{1}, y_{1}\right\rangle & \cdots & \left\langle x_{1}, y_{n}\right\rangle \\
\vdots & \ddots & \vdots \\
\left\langle x_{n}, y_{1}\right\rangle & \cdots & \left\langle x_{n}, y_{n}\right\rangle
\end{array}\right|}{\left\|y_{1}, \ldots, y_{n}\right\|^{S}} \leq\left\|x_{1}, \ldots, x_{n}\right\|^{E} . \\
\Rightarrow\left\|x_{1}, \ldots, x_{n}\right\|^{F} \leq\left\|x_{1}, \ldots, x_{n}\right\|^{E} .
\end{gathered}
$$

This completes the proof.

Corollary 3.1. $\|, \ldots, .,\|^{G},\|, \ldots,\|^{S},\|, \ldots, .\|^{D},\|, \ldots, .\|^{E}$ and $\|, \ldots, .\|^{F}$ are identical.

Proposition 3.3.0n a separable Hilbert space $X,\|, \ldots,\|^{F}$ and $\|, \ldots,\|_{2}$ are identical.

Proof: Let $\left\{e_{1}, e_{2}, \ldots\right\}$ be a complete orthonormal set in $X$.Then, $\|, \ldots,\|_{2}$ may be derived directly from standard n-norm $\|, \ldots,\|^{s}[6] \Rightarrow\|, \ldots,\|^{s}$ and $\|, \ldots,\|_{2}$ are identical. Also, $\|, \ldots,\|^{S}$ and $\|, \ldots, .,\|^{E}$ are identical. So, $\|, \ldots,\|^{E}$ and $\|, \ldots,\|_{2}$ are identical. But, $\|, \ldots, .,\|^{E}$ and $\|, \ldots,\|^{F}$ are identical [proposition 3.2]. Therefore, $\|, \ldots, .,\|^{F}$ and $\|, \ldots,\|_{2}$ are identical.

Corollary 3.2.0n a separable Hilbert space $X,\|, \ldots,\|^{G},\|, \ldots,\|^{S},\|, \ldots, .,\|^{D},\|, \ldots, .\|^{E},\|, \ldots, .,\|_{2}$ and $\|, \ldots,\|^{F}$ are identical.

\section{References}

[1] Gähler, S. (1964). Lineare 2-normierter räume. Math.Nachr., 28(1), 43.

[2] Gunawan, H., Gozali, S. M., \& Neswan, O. (2010). On n-norms and bounded n-linear functionals in a Hilbert space. Ann. Funct. Anal., 1(1), 72-79

[3] Gunawan, H. (2001). The space of p-summable sequences and it's natural n-norm bull. Austral. Math. Soc., 64, 137-147.

[4] Singh, M. P., \& Romen, S. (2014, May). On a new n-norm and some identical n-norms on a Hilbert space. IJSER, 5(5), 995-999

[5] Gähler, S. (1969). Untersuchungenüberverallgemeinerte m-metrischeräume I. Math. Nachr., 40, 165-189.

[6] Gähler, S. (1969). Untersuchungenüberverallgemeinerte m-metrischeräume II. Math. Nachr., 40, 229-264.

[7] Gähler, S. (1970). Untersuchungenüberverallgemeinerte m-metrischeräume III. Math. Nachr., 41, 23-36.

[8] Gunawan, H. (2002). Inner products on n-inner product spaces. Soochow J. Math, 28, 389-398.

[9] Misiak, A. (1989). n-inner product spaces. Math. Nachr., 140, 299-319.

[10] Gunawan, H., \& Mashadi. (2001). On n-normed spaces. Int. J. Math. Math. Sci., 27, 631-639.

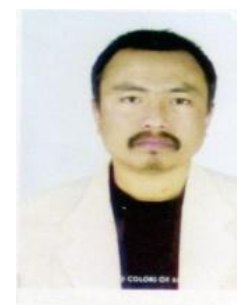

M. P. Singh was born at Thamnapokpi, Manipur, India. He studied the masters of science in mathematics from Delhi University, Delhi in 1997. He got JRF from CSIR in 1997 and he got PhD in mathematics from Manipur University in 2013.

He is working as an assistant Professor in the Department of Mathematics, Manipur University, India. He has published one book Slant Hankel Operators, Scholars Press, 2015. He has also published recently the papers "On a new norm and some identical n-norms on a Hilbert space," IJSR, vol. 5, issue 5, 2014 and “Identicalness of n-norms," Adv. Ineq. Appl., 2014. 